\title{
Effet des téguments de fèves de cacao sur la fertilité chimique d'un ferralsol et quelques paramètres de croissance du manioc, à Ahoué, Sud-Est Côte d'Ivoire
}

\author{
Kouadio Koffi Patrice*, Yoboué Kouadio Emile, Touré Nantarie, Beugré Christian Moise, Aka Kacou \\ Félix, Kouakou Kouassi Josselin et Yao-Kouamé Albert \\ Université Félix Houphouët-Boigny d'Abidjan-Cocody, UFR des Sciences de la Terre et des Ressources Minières \\ (STRM), Département des Sciences du Sol, 22 BP 582 Abidjan 22, Côte d'Ivoire \\ *Auteur correspondant : kkpatrice@hotmail.fr
}

Original submitted in on $1^{\text {st }}$ November 2017. Published online at www.m.elewa.org on $31^{\text {st }}$ January 2018 https://dx.doi.org/10.4314/jab.v121i1.5

\section{RESUME}

Objectif : utiliser les engrais organiques à base de téguments des fèves de cacao pour une gestion durable des ferralsols.

Méthodologie et résultats: Dans un dispositif expérimental en bloc de Fisher, trois traitements des téguments de fèves de cacao T1 (10 tha $\left.{ }^{-1}\right)$, T2 (15 tha- $\left.{ }^{-1}\right)$, T3 $\left(20\right.$ t.ha $\left.^{-1}\right)$ et un témoin T0 $\left(0\right.$ t.ha $\left.^{-1}\right)$ ont été mis en place avec quatre répétitions à Ahoué, dans le Sud-Est de la Côte d'lvoire. L'analyse des téguments de la fève de cacao indique de fortes teneurs en phosphore total $\left(7600 \mathrm{mg} \cdot \mathrm{kg}^{-1}\right)$, en potassium (797,95 Cmol.kg-1), en calcium (19,21 Cmol.kg-1), en magnésium (234,47 Cmol. $\left.\mathrm{kg}^{-1}\right)$ en carbone total (320 g. $\left.\mathrm{kg}^{-1}\right)$ et en azote total $\left(18 \mathrm{~g} . \mathrm{kg}^{-1}\right)$ avec un rapport C/N égal à 17,78. Les principaux résultats montrent que l'apport des téguments a eu des effets positifs sur l'ensemble des caractéristiques chimiques du sol et des paramètres de croissance du manioc. Les valeurs obtenues $\mathrm{du} \mathrm{pH}$ de la parcelle Témoin T0 et des parcelles traitées avec T1, T2, T3 indiquent, respectivement, $4 ; 5,4 ; 5,5$ et 5,8. Les rapports $\mathrm{C} / \mathrm{N}$ de la parcelle Témoin T0 et des parcelles traitées avec T1, T2, T3 donnent, respectivement, 24,33; 12,83; 12,36 et 12,16. S'agissant des teneurs de la CEC, les valeurs obtenues indiquent : 2,$32 ; 7,2 ; 11,44$ et $12,5 \mathrm{cmol} . \mathrm{kg}-1$, respectivement, pour les traitements T0, T1, T2 et T3. Le plus petit indice et les petites tailles des plants de manioc ont été observés sur les parcelles témoin, respectivement, après 71 et 78 jours de plantation et par contre, le plus grand indice de vigueur et les plus grandes tailles ont été observés sur les parcelles fertilisées avec le traitement T3.

Conclusion et application: L'étude précise que l'incorporation de doses croissantes de téguments au sol influence l'ensemble des caractéristiques chimiques du sol et des paramètres de croissance du manioc. Les meilleurs résultats ont été obtenus à la dose de 20 t.ha $^{-1}$ des téguments de fèves de cacao. Ce travail de recherche agronomique montre l'importance des téguments de fèves de cacao sur la fertilité chimique des ferralsols en vue d'accroître durablement les productions et préserver l'environnement. La détermination de la dose optimale et de l'effet combiné de ces téguments avec les engrais minéraux sur les sols pourrait être envisagée pour une gestion durable des différents types de sols en Côte d'Ivoire.

Mots clés : Téguments de fève de cacao, fertilité chimique, Ferralsol, Manioc, Ahoué, Côte d'Ivoire 


\section{ABSTRACT}

Objective: use organic fertilizers based on cocoa beans teguments for sustainable management of ferralsols.

Methodology and results: In an experimental block design of Fisher, Three treatments of Cocoa bean teguments: T1 (10 t.ha-1 $)$, T2 (15 t.ha-1), T3 (20 tha- $\left.{ }^{-1}\right)$ and one Control T0 were set up with four repetitions in Ahoué, South-East of Côte d'Ivoire. The analysis of the Cocoa bean teguments indicates high levels of total phosphorus (7600 mg.kg-1), in potassium (797.95 Cmol. $\left.\mathrm{kg}^{-1}\right)$, in calcium $\left(19.21 \mathrm{Cmol}^{-1} \mathrm{~kg}^{-1}\right)$, in magnesium (234.47 Cmol. $\left.\mathrm{kg}^{-1}\right)$, in total carbon $\left(320 \mathrm{~g}^{\mathrm{kgg}}{ }^{-1}\right)$ and in total nitrogen $\left(18 \mathrm{~g}^{\mathrm{kg}}{ }^{-1}\right)$ with a report $\mathrm{C} / \mathrm{N}$ which gives 17.78. The main results show that the contribution of the teguments had positive effects on the set of the chemical characteristics of the soil and on the parameters of growth of the cassava. The values obtained from the $\mathrm{pH}$ of the witness soil (TO) and the handled plots of land T1, T2, T3 indicates, respectively $4.2 ; 5.4 ; 5.5$ and 5.8 . The reports $\mathrm{C} / \mathrm{N}$ of the witness soil and the handled plots of land $\mathrm{T} 1, \mathrm{~T} 2$, T3, give, respectively, 24.33; 12.83; 12.36 and 12.16. As regards the contents of the CEC, the values obtained give: 2.32; 7.2; 11.44 and $12.5 \mathrm{cmol}_{\mathrm{kg}}{ }^{-1}$, respectively, for T0, T1, T2 and T3 treatments. The lowest index and small sizes of cassava plants were observed on the control plots, respectively, after 71 and 78 days of planting and against the greatest vigor index and largest sizes were observed on plots fertilized with $\mathrm{T} 3$ treatment.

Conclusion and application: The study states that the incorporation of increasing doses of Cocoa bean teguments influences all of the soil's chemical characteristics and cassava parameters. The best results were obtained at a dose of 20 t.ha-1 $^{-1}$ of cocoa bean teguments. This agronomic research shows the importance of cocoa bean teguments on the chemical fertility of ferralsols with a view to sustainably increasing production and preserving the environment. Determination of the optimum dose and the combined effect of these teguments with mineral fertilizers on soils could be considered for sustainable management of different soils in Côte d'Ivoire.

Key words: Cocoa bean teguments, chemical fertility, Ferralsol, Cassava, Ahoué, Côte d'Ivoire.

\section{INTRODUCTION}

La dégradation des ressources naturelles demeure un problème majeur pour le développement agrosylvo-pastoral de l'Afrique de l'Ouest et particulièrement de la Côte d'Ivoire où les sols font l'objet d'une exploitation agricole depuis plus de 50. Les éléments nutritifs exportés lors des cultures ne sont pas remplacés de manière adéquate (FAO, 2003). En Côte d'Ivoire, la place prépondérante occupée par l'agriculture dans l'économie oblige les paysans à une pratique de cultures intensives à cause de la forte pression sur les terres, due aux besoins croissants engendrés par l'accroissement démographique, et au manque de terres cultivables (N'Goran et al., 1997). La conséquence de cette pression est la diminution des jachères, conduisant à la dégradation du sol et aux faibles rendements des cultures (Yemefack et Nounamo, 2000). Le recours à la fertilisation constitue le moyen le plus approprié pour apporter aux végétaux cultivés le complément indispensable à l'offre du sol. La fertilisation minérale ou organique a pour but essentiel de satisfaire les besoins nutritifs des plantes en vue de leur assurer une croissance et un développement optimum. L'utilisation intensive des engrais chimiques de synthèse présente un certain nombre d'inconvénients ; la fertilisation, privilégiant les engrais minéraux aux dépens de la fumure organique augmente certes, le rendement immédiat, mais déstructure progressivement le sol (Cattan et al., 2001). Son utilisation exclusive entraîne une augmentation de l'acidité, une dégradation du statut physique et une baisse du taux de matière organique du sol (Boli et Roose, 2000). Les problématiques de dégradation des sols ont fait l'objet de nombreux travaux de recherche, mais, il convient de constater que ces problèmes restent toujours d'actualité et sans réponse (Angui et al., 2005). De nombreux travaux ont montré que les amendements organiques 
jouent un rôle important sur diverses propriétés du sol (Djéké et al., 2011). Ayanlaja et Sanwo (1991) ont montré que la décomposition des résidus végétaux permet d'améliorer considérablement le niveau des nutriments et la matière organique dans les sols. Au regard de tous ces travaux, la fertilisation organique pourrait jouer un rôle important sur diverses propriétés du sol,

\section{MATERIEL ET METHODES}

Description du site : Située au Sud-Est de la Côte d'Ivoire, la zone d'étude (Ahoué) est délimitée par les coordonnées $05^{\circ} 30^{\prime} 43^{\prime \prime} \mathrm{N}$ et $03^{\circ} 56^{\prime} 31^{\prime \prime} \mathrm{W}$ (Figure 1). Le climat de cette localité est de type subéquatorial à quatre saisons, avec une pluviométrie et des températures moyennes annuelles de $2000 \mathrm{~mm}$ et $26^{\circ} \mathrm{C}$ (Kouakou et al., 2016). La zone appartient au permettant de justifier leur utilisation (Sangaré et al., 2001). C'est dans ce cadre que sont menées, à Ahoué, au Sud-Est de la Côte d'Ivoire, des expérimentations avec le fumier des téguments de fèves de cacao en vue d'évaluer leur effet sur la fertilité chimique du sol et des paramètres de croissance du manioc.

secteur ombrophile du domaine guinéen, caractérisé par la forêt dense humide sempervirente. Le relief se présente comme un modelé en demi-orange (dômes). Cette zone est caractérisée par un relief constitué de vastes plaines (alluviales) et de bas plateaux parcourus par une succession de vallonnements.

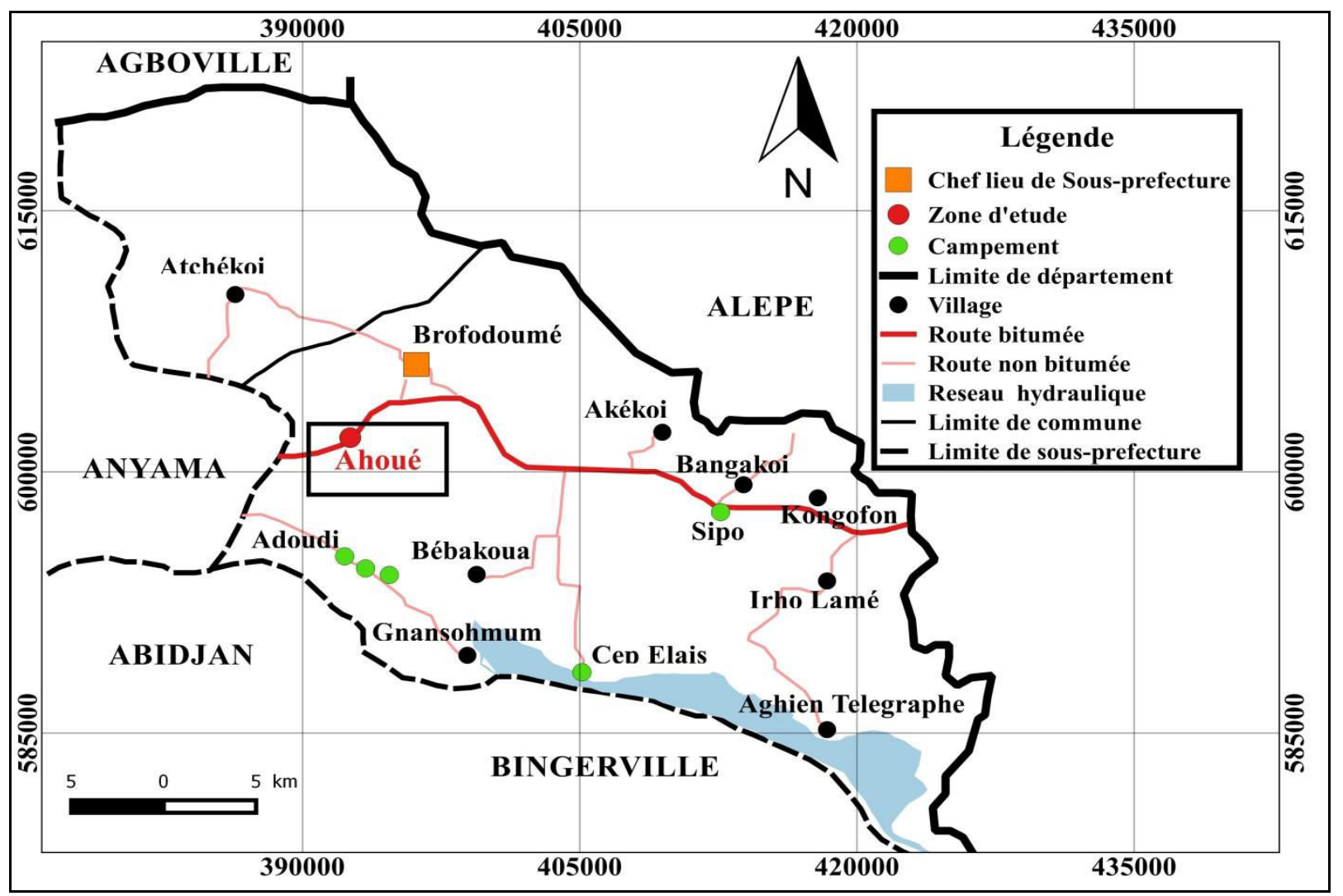

Figure 1 : Localisation du site d'étude

Matériel végétal : Les essais conduits portent sur une des cultures vivrières de cette région. II s'agit du manioc, plante à racines tubérisées. La variété de manioc (Manihot esculenta Crantz) utilisée pour cette étude est Improved. African Cassava (Figure 2). C'est une variété résistante, couramment cultivée et consommée par la population ivoirienne sous différentes formes (attiéké, pâte de manioc, placali, foutou, toh, farine, amidon, gari, pain, gâteau). 


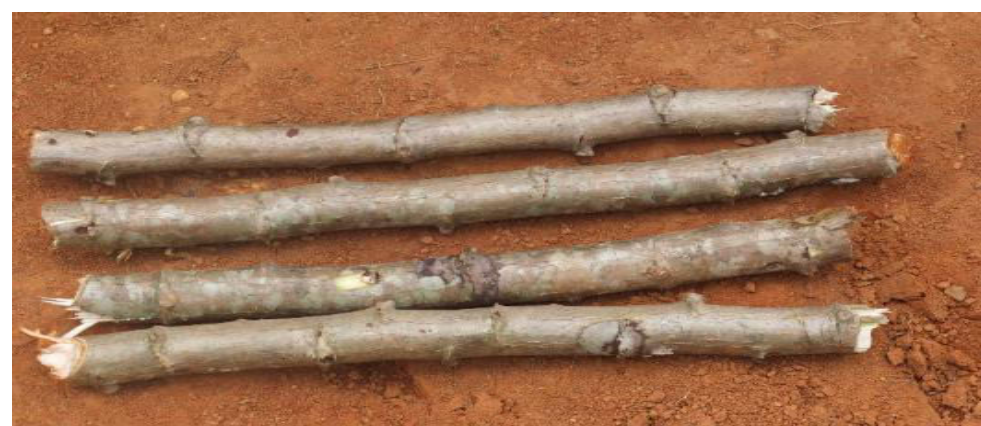

Figure 2 : Boutures de manioc (Improved African Cassava communément appelé « Yacé »)

Matériel fertilisant : La fumure organique utilisée est constituée de téguments de fèves de cacao (Figure 3). Ce matériel organique fertilisant provenait d'un dépotoir, lieu de commerce des téguments de fèves de cacao compostés dans la commune d'Abobo (Abidjan, Côte d'Ivoire).

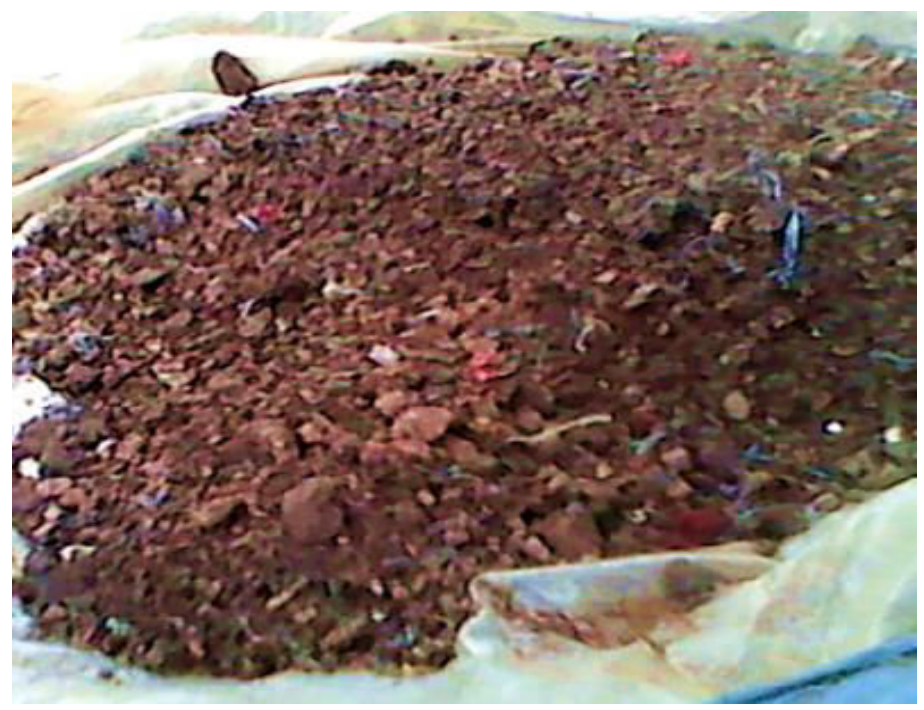

Figure 3 : Téguments de fèves de cacao

\section{METHODES}

Dispositif expérimental : Des essais agronomiques sont mis en place, en milieu paysan, selon un dispositif en blocs randomisés ou blocs de "Fisher » avec quatre répétitions (Figure 4). Les microparcelles de $9 \mathrm{~m}^{2}(3 \mathrm{~m} \times 3 \mathrm{~m})$ sont numérotées de T0 à T3 et les blocs sont des répétitions. Dans le cas d'expérience réalisée sur le terrain, les blocs constituent un ensemble de parcelles voisines et très semblables des unes des autres. La répartition des objets au sein des différents blocs se fait normalement de façon complètement aléatoire et indépendamment d'un bloc à l'autre. Les téguments de fèves de cacao constituent le facteur étudié à quatre (4) niveaux : T1 (10 tha-1), T2 (15 tha-1), T3 $\left(20\right.$ t.ha $\left.^{-1}\right)$ et TO $\left(0\right.$ t.ha $\left.^{-1}\right)$. Les blocs sont séparées de $2 \mathrm{~m}$ et les sous parcelles sont séparées entre elles de $1 \mathrm{~m}$. Ces différentes doses (traitements) sont apportées lors de la préparation du sol ou à la plantation. 
$15 \mathrm{~m}$

BLOC1

BLOC2

BLOC4

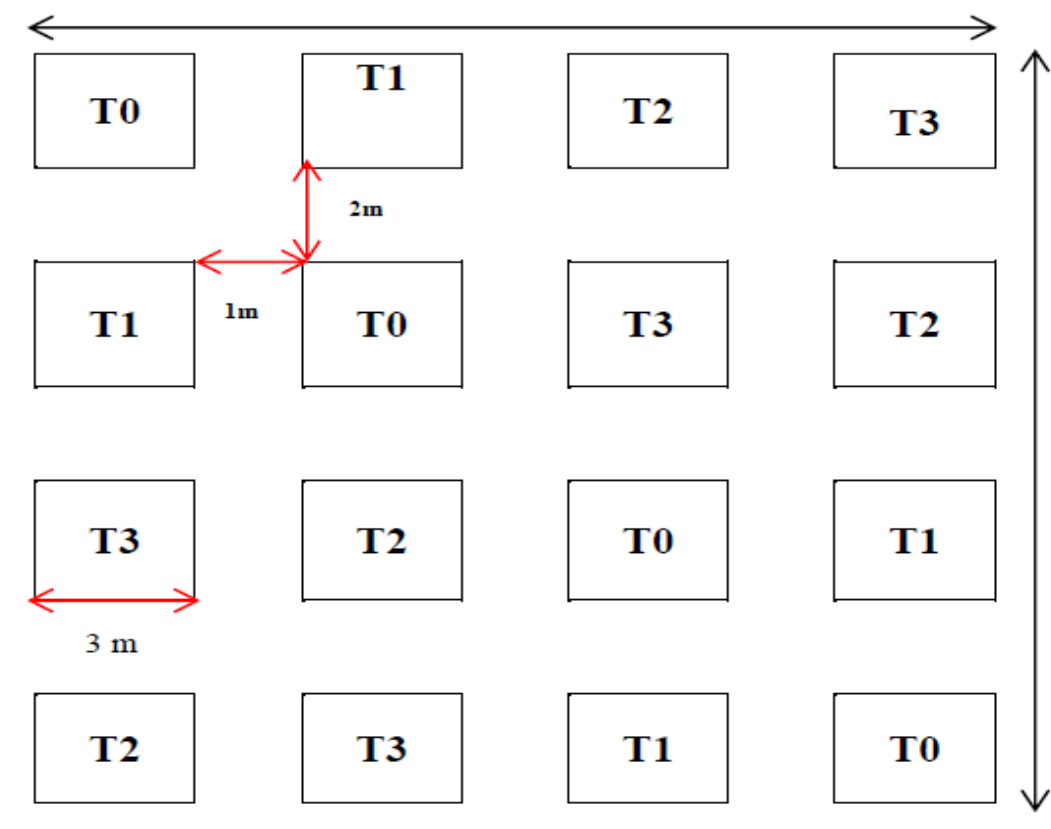

$18 \mathrm{~m}$

Figure 4 : Dispositif expérimental

\section{Mise en place des essais}

Préparation du sol et application du matériel organique fertilisant: La préparation du sol a été réalisée mécaniquement. Les téguments ont été pesés puis appliqués en une mince couche régulière selon les différentes doses sur chacune des micro-parcelles concernées, suivant le dispositif expérimental. Ils ont été manuellement enfouis à la daba.

Mise en culture: Le mode de plantation est celui utilisé par les directions des programmes de recherche et de l'appui au développement et des systèmes d'information CNRA (N'zué et al., 2005). Des boutures de 20 à $30 \mathrm{~cm}$ de long (4 à 6 nœuds) ont été prélevées sur des tiges saines âgées d'au moins 6 mois. Les billons ont été confectionnés selon les pratiques locales. La densité de plantation s'élève à 10000 plants par hectare (1 m entre les lignes et $1 \mathrm{~m}$ entre les plants sur la même ligne), soit 16 boutures de manioc ont été disposées sur chaque micro parcelle de dimension $9 \mathrm{~m}^{2}$.
Collecte des échantillons de téguments et du sol Échantillon de téguments de fèves de cacao:Les téguments de fèves de cacao ont été obtenus dans un dépotoir à Abobo (Abidjan). C'est un lieu de commerce des téguments de fèves de cacao compostés. Ces produits ont ensuite été séchés, broyés et tamisés $(\varnothing<$ $2 \mathrm{~mm}$ ) avant d'être épandus (Figure 5).

Échantillons de sol: L'échantillonnage s'est déroulé dans la couche de $0-30 \mathrm{~cm}$ à cause de la profondeur d'enracinement du manioc pouvant aller jusqu'à plus de $30 \mathrm{~cm}$ (Hauser et al., 2013) et aussi de certains travaux passés (Akanza et Yao-Kouamé, 2011). Au sein de chaque parcelle élémentaire, un prélèvement aléatoire de sol a été effectué en cinq points différents, en suivant les deux principales diagonales. Le premier, intervenu avant les apports des téguments, visait à déterminer le potentiel brut du sol en éléments fertilisants et le second, intervenu après la récolte. 


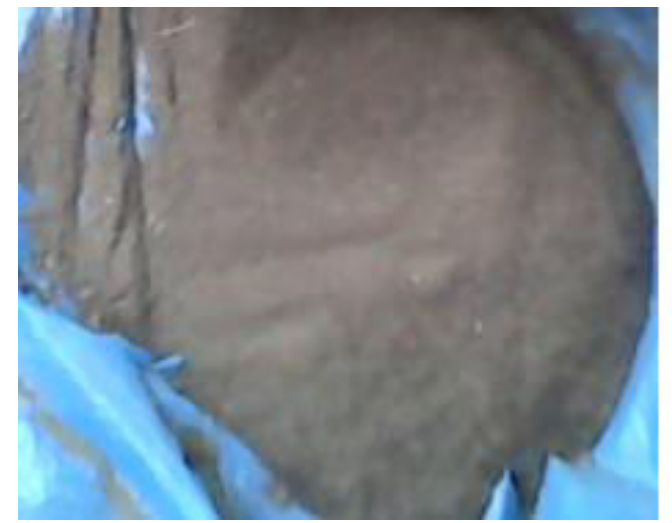

Figure 5 : Téguments de fèves de cacao broyés

Analyse chimique des téguments et du sol: Les mesures du pH eau, pour évaluer l'acidité du sol, ont été effectuées dans une suspension de sol dans l'eau à l'aide d'un $\mathrm{pH}$-mètre dans un rapport 1/2,5. La méthode à l'acétate d'ammonium $1 \mathrm{M}$ à $\mathrm{pH} 7$ (Anderson et al., 1993) a été utilisée pour déterminer la Capacité d'Échange des Cations (CEC) et les teneurs en bases échangeables $\left(\mathrm{Ca}^{2+}, \mathrm{Mg}^{2+}, \mathrm{K}^{+}\right)$. Le carbone organique total a été déterminé selon la méthode de WalkleyBlack (1934). La détermination des teneurs du sol en azote total (N) s'est faite par la méthode de Kjeldahl de Rittember (Bremner et Mulvaney, 1982) et celles du phosphore total et assimilable, respectivement, par colorimétrie en présence de molybdate d'ammonium et d'acide ascorbique après extraction par attaque à l'acide perchlorique (Olsen et Sommers, 1982), et par colorimétrie après extraction par une solution alcaline de bicarbonate de sodium $\left(\mathrm{NH}_{4} \mathrm{~F} 0,5 \mathrm{~N}+\mathrm{NaHCO}_{3} 0,5\right.$ $\mathrm{N}, \mathrm{pH} 8,5)$ (Dabin, 1967).

Paramètres agronomiques du manioc mesurés

- Taux de levée: Le taux de levée, paramètre très important en agronomie, permet d'apprécier le pourcentage de reprise des boutures de manioc après dix jours de plantation (Egle, 2012). Les pieds levés ont été comptés sur chaque sous parcelle par bloc. Les observations ont débuté deux semaines après la

\section{RESULTATS}

Caractéristiques morpho-pédologiques des sols du site: Les fosses pédologiques ouvertes, géoréférencées, décrites ont fait l'objet de cette étude (Figure 6), Les différents horizons sont en général, peu humifères, humides, poreux avec peu de racines orientées de façon subhorizontale Ce sol présente une structure particulaire à sous structure grumeleuse, qui plantation. Après la deuxième observation, une troisième a eu lieu à la quatrième semaine, en vue de recenser les éventuelles plantules en retard au niveau de la levée (Bakayoko et al., 2013).

- Indice de vigueur : L'indice de vigueur $(X)$, caractère important du développement des plants (Berchoux et Lecoustre, 1986), a été déterminé deux mois après plantation, selon la formule suivante:

$\left.X=\log \left[\left(C^{2} \times H\right) / 4 \pi\right)\right](01)$ où $C=$ circonférence au collet, $\mathrm{H}=$ hauteur $(\mathrm{H}$ et $\mathrm{C}$ sont exprimées en centimètre).

- Hauteur de plants: Chaque semaine après deux mois de plantation, dix plantes prises comme caractéristiques dans chaque sous parcelle ont servi de mesure de la hauteur La hauteur a été mesurée à l'aide d'un ruban mètre. Les mesures ont été prises en centimètre, et ont été consignées sur une fiche technique.

Analyse Statistique des données: Les données recueillies ont été soumises à une analyse de variance (ANOVA) à l'aide du logiciel SAS. Le test de NewmanKeuls a été utilisé pour la comparaison des moyennes lorsque des différences significatives entre les traitements ont été révélées au seuil de probabilité de $5 \%$.

devient fragmentaire polyédrique à partir de $20 / 22 \mathrm{~cm}$ de profondeur. Ces horizons présentent une texture sablo-limoneuse dans les horizons de surface, puis argilo-sableuse à partir de $60 \mathrm{~cm}$. La couleur du sol passe de brun à jaune rougeâtre à partir de $60 \mathrm{~cm}$. Les horizons sont généralement peu cohérents avec un drainage relativement bon. 


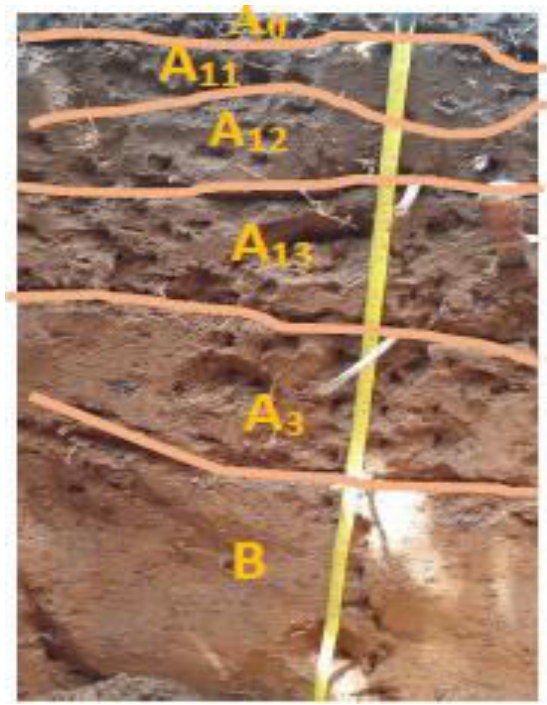

Figure 6 : Profil AP3

Caractéristiques chimiques des téguments: total $\left(320 \mathrm{~g} \cdot \mathrm{kg}^{-1}\right)$, en azote total $\left(18 \mathrm{~g} \cdot \mathrm{kg}^{-1}\right)$, en L'analyse des téguments de fèves de cacao montre que la composition chimique, déterminée à partir de la Phosphore total $(7600 \mathrm{mg} . \mathrm{kg})$, en Potassium $(797,95$ matière sèche, indique des fortes teneurs en Carbone $\left.\mathrm{Cmol.kg}^{-1}\right)$, en Calcium $\left(19,21 \mathrm{Cmol}^{\mathrm{kg}}{ }^{-1)}\right.$ et en Magnésium (234,47 Cmol.kg-1) (Tableau 1).

Tableau 1 : Caractéristiques chimiques des téguments de fèves de cacao broyés

\begin{tabular}{l|c}
\hline Caractéristiques chimiques & Téguments de fèves de cacao \\
\hline $\mathrm{C}\left(\mathrm{g} \cdot \mathrm{kg}^{-1}\right)$ & 320 \\
$\mathrm{~N}\left(\mathrm{~g} \cdot \mathrm{kg}^{-1}\right)$ & 18 \\
$\mathrm{C} / \mathrm{N}$ & 17,78 \\
Phosphore total $\left(\mathrm{mg} \cdot \mathrm{kg}^{-1}\right)$ & 7600 \\
$\mathrm{Ca}\left(\mathrm{Cmol} . \mathrm{kg}^{-1}\right)$ & 19,21 \\
$\mathrm{Mg}\left(\mathrm{Cmol} . \mathrm{kg}^{-1}\right)$ & 234,47 \\
$\mathrm{~K}\left(\mathrm{Cmol} . \mathrm{kg}^{-1}\right)$ & 797,95 \\
$\mathrm{Na}\left(\mathrm{Cmol} . \mathrm{kg}^{-1}\right)$ & 8,69 \\
\hline
\end{tabular}

Caractéristiques chimiques du sol du site avant l'implantation des essais: Les caractéristiques chimiques du sol du site avant l'implantation des essais ont été déterminées (Tableau 2). Elles témoignent d'une faible fertilité au regard des faibles teneurs en éléments organiques $\left(\mathrm{N}=0,3 \mathrm{~g} \cdot \mathrm{kg}^{-1}\right.$ et $\left.\mathrm{C}=7,3 \mathrm{~g} \cdot \mathrm{kg}^{-1}\right)$ mal décomposés $(\mathrm{C} / \mathrm{N}=24,33)$. Le potentiel de fixation et d'échange d'éléments nutritifs du complexe argilohumique reste faible au regard des valeurs de la somme des bases échangeables $\left(S=0,23 \mathrm{cmol} . \mathrm{kg}^{-1}\right)$ et du taux de saturation en bases ( $V=9,91 \%$ ). Sa capacité d'échange cationique reste également faible $\left(C E C=3,10 \mathrm{cmol}^{\mathrm{kg}}{ }^{-1}\right)$ ainsi que sa teneur en phosphore assimilable $\left(85 \mathrm{mg}^{\mathrm{kg}}{ }^{-1}\right)$.

Tableau 2: Caractérisation chimique du sol du site avant l'implantation des essais.

\begin{tabular}{|c|c|c|c|c|c|c|c|c|c|c|c|}
\hline pH eau & $\begin{array}{l}N \\
(g\end{array}$ & ${ }^{-1}$ C & $\mathrm{C} / \mathrm{N}$ & $\begin{array}{c}\text { Pass. } \\
\left(\mathrm{mg.kg}^{-1}\right)\end{array}$ & $\mathrm{Ca}$ & $\mathrm{Mg}$ & $\begin{array}{c}\mathrm{K} \\
\left(\mathrm{cmol}^{2} \mathrm{~kg}^{-1}\right)\end{array}$ & $\mathrm{Na}$ & CEC & $S$ & $\begin{array}{c}V \\
(\%)\end{array}$ \\
\hline 4,2 & 0,3 & 7,3 & 24,33 & 85 & 0,064 & 0,135 & 0,013 & 0,018 & 2,32 & 0,23 & 9,91 \\
\hline
\end{tabular}

Pass. $=$ Phosphore assimilable $; \mathrm{S}=$ Sommes des bases $; \mathrm{V}=$ Taux de saturation 
Effets des différents traitements sur des Ainsi, avec les traitements T1, T2 et T3, les valeurs composantes chimiques du sol

pH eau du sol : Les parcelles fertilisées avec T1, T2 et $\mathrm{T} 3$ ont connu une évolution de leur $\mathrm{pH}$ eau par rapport aux parcelles témoin dont le $\mathrm{pH}$ eau n'a pas évolué (Figure 6). de $\mathrm{pH}$ eau donnent, respectivement, 5,$2 ; 5,5$ et 5,8 et quant au sol témoin, le pH eau indique 4 .

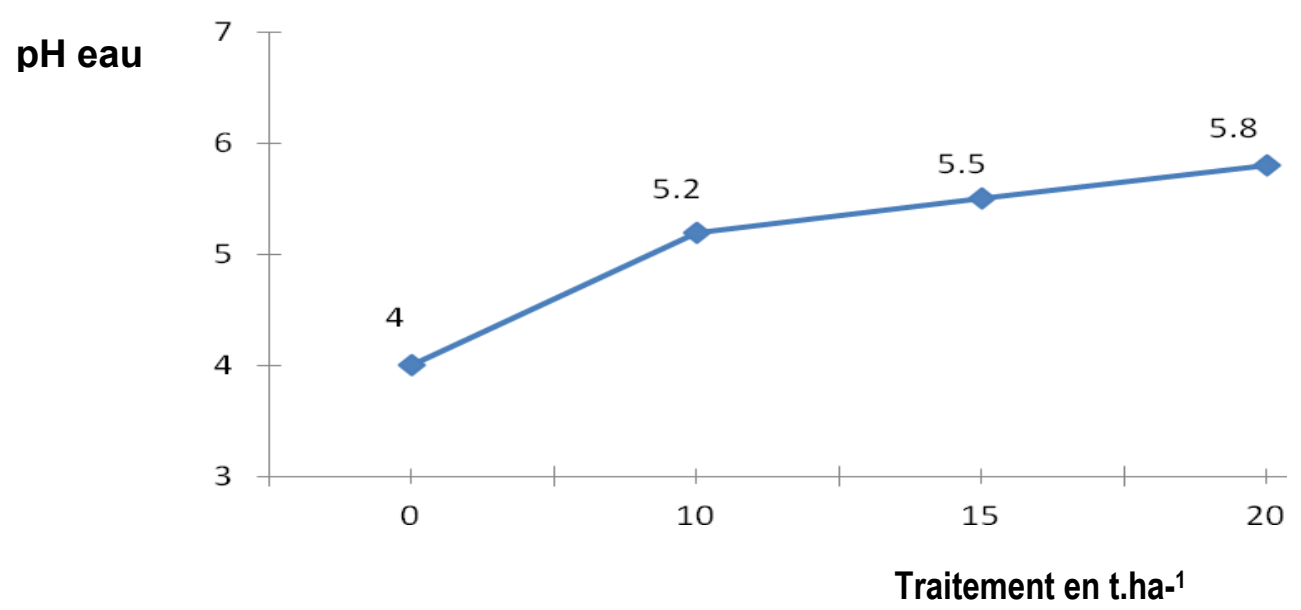

Figure 6 : Évolution du pH eau

Rapport $\mathrm{C} / \mathrm{N}$ : La parcelle témoin a un rapport $\mathrm{C} / \mathrm{N}$ plus élevé que celles qui ont reçu les doses croissantes des téguments de fèves de cacao. Le rapport $\mathrm{C} / \mathrm{N}$ des parcelles qui ont reçu le T3, est plus faible $(12,2)$ que celles fertilisées par les traitements T1, T2 et le témoin TO dont les rapports $\mathrm{C} / \mathrm{N}$ donnent, respectivement, 12,$8 ; 12,4$ et 24,3 (Figure 7).

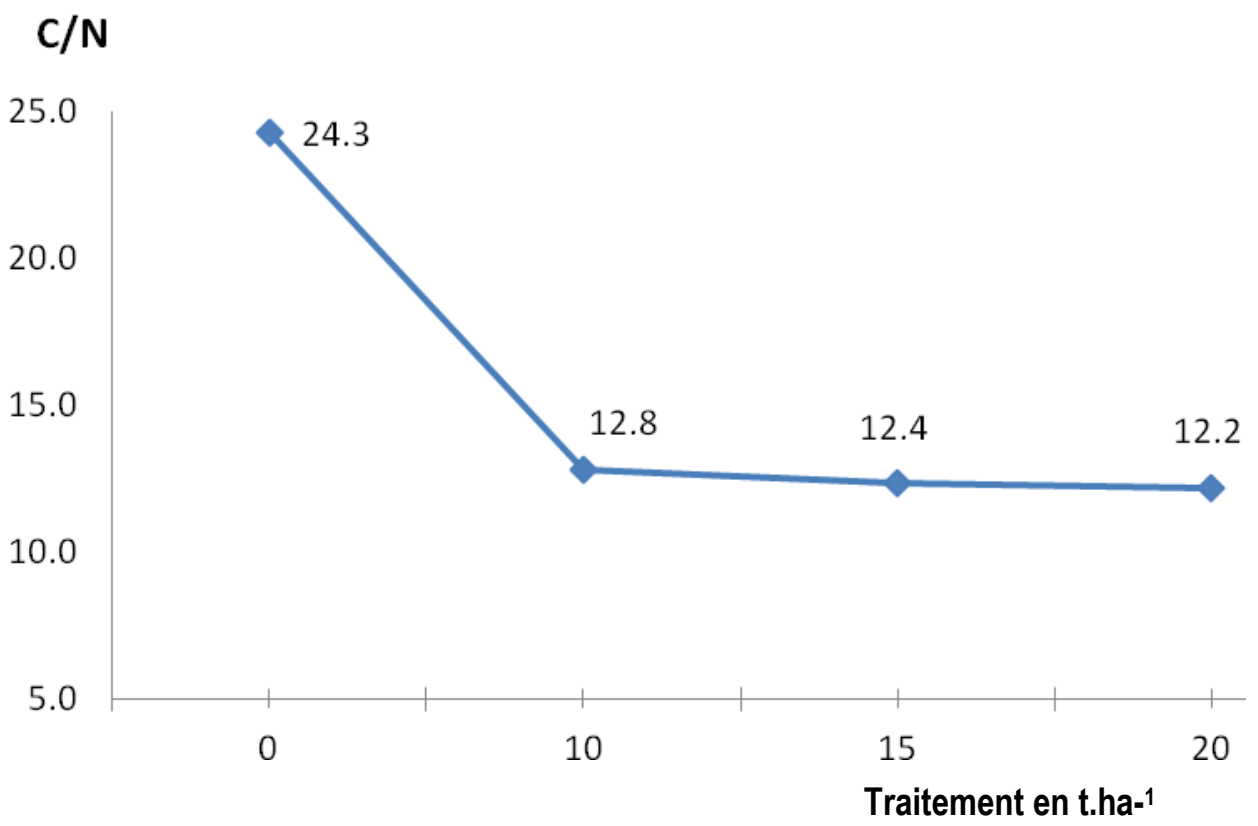

Figure 7 : Évolution du rapport $\mathrm{C} / \mathrm{N}$ 
Capacité d'Échange Cationique (CEC) et Somme des bases échangeables (S): Les traitements T1, T2 et T3 ont influencé positivement les CEC des parcelles amendées et cela, en fonction des doses croissantes des téguments de fèves apportées (Figure 8). Le traitement T3 présente la CEC la plus élevée (12,5 $\mathrm{cmol}^{\mathrm{kg}}{ }^{-1}$ ) par rapport aux traitements T1, T2 et au témoin TO, dont les teneurs des CEC sont, respectivement, égales à $7,2 \mathrm{cmol} . \mathrm{kg}^{-1}, 11,44 \mathrm{cmol}^{\mathrm{kg}} \mathrm{kg}^{-1}$ et $2,3 \mathrm{cmol}^{\mathrm{kg}}{ }^{-1}$. S'agissant la sommes des bases échangeables (S), les traitements $\mathrm{T} 1, \mathrm{~T} 2$ et $\mathrm{T} 3$ ont eu un effet positif. Les traitements T1, T2 et T3 présentent des bases échangeables plus élevées, respectivement, égales à $4,5,7,3$ et $10,5 \mathrm{cmol}^{\mathrm{kg}}{ }^{-1}$ par rapport au traitement témoin dont la valeur de $\mathrm{S}$ est égale à $0,23 \mathrm{cmol}_{\text {.kg-1 }}$ (Figure 8).

\section{CEC et S}

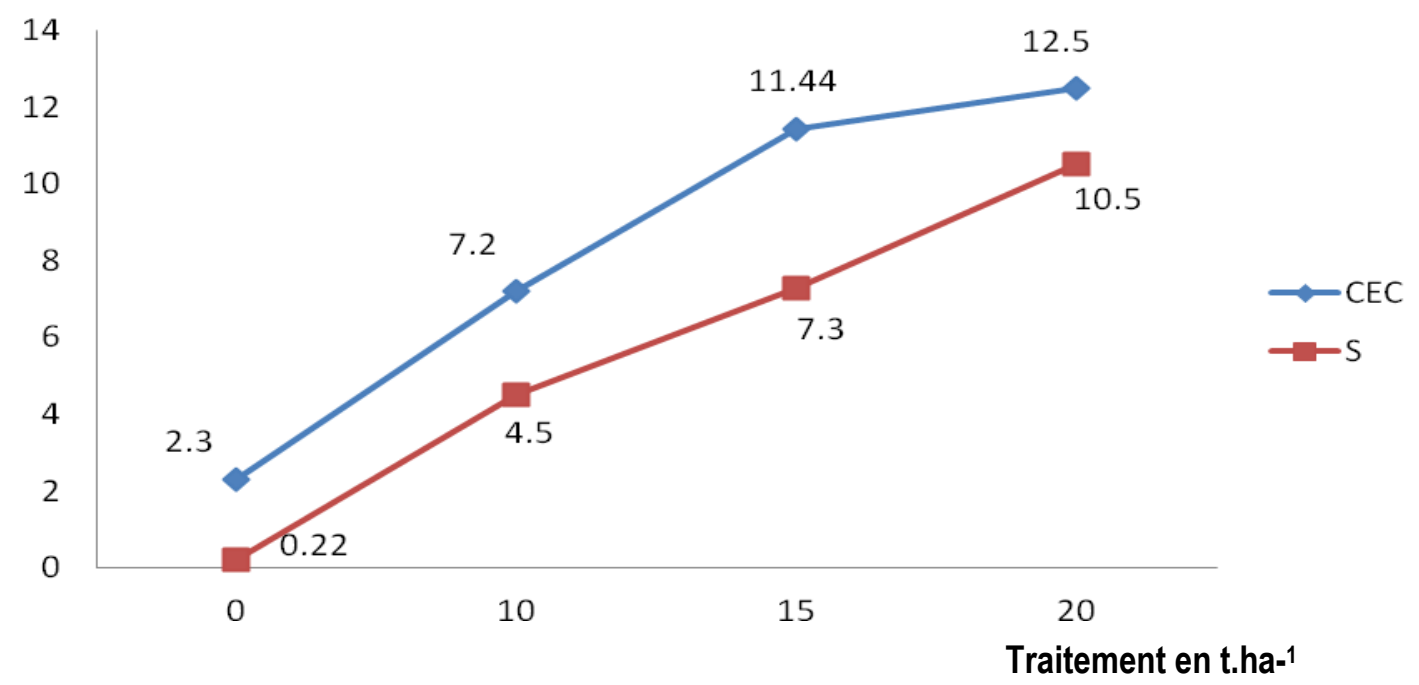

Figure 8 : Évolution de la CEC et de S

Taux de saturation du complexe adsorbant (V) : Le taux de saturation du complexe adsorbant est plus élevé dans les parcelles qui ont reçu les fertilisants organiques que celui de la parcelle témoin (Figure 9).
Ces taux ont varié de 62,5\% (T1) à $84 \%$ (T3) pour les parcelles fertilisées et quant aux parcelles témoin, ce taux de saturation du complexe est resté très faible (Figure 9).

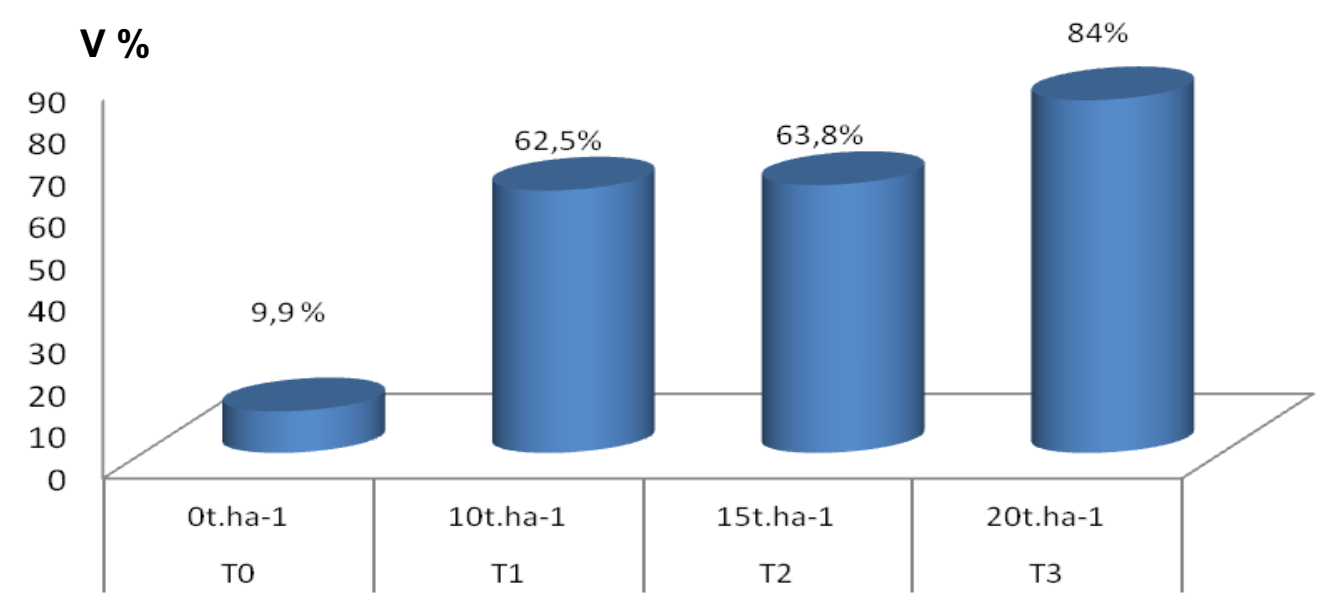

Figure 9 : Évolution du Taux de saturation du complexe adsorbant (V)

Traitements 
Kouadio et al., J. Appl. Biosci. 2018 Effet des téguments de fèves de cacao sur la fertilité chimique d'un ferralsol et quelques paramètres de croissance du manioc, à Ahoué, Sud-Est Côte d'Ivoire

Effets des téguments de fèves de cacao sur des paramètres de croissance du manioc

Taux de levée: Après 14 jours de plantation, le taux de levée dans chacune des sous parcelles amendées avec l'engrais organique est supérieur à $50 \%$. Toutefois, une légère amélioration du taux de levée $(59,75 \%)$ est observée dans les sous parcelles

fertilisées avec le traitement $\mathrm{T} 1$. Ce taux de levée après 21 et 28 jours de plantation, varie nettement pour chaque traitement, mais, avec une légère amélioration dans les sous parcelles T2 et T3 (82,8 à $87,5 \%)$ (Tableau 3).

Tableau 3 : Taux de levée

\begin{tabular}{l|c|c|c|c}
\hline \multirow{2}{*}{ Traitements } & \multicolumn{3}{|c|}{ Taux de levée } & \multirow{2}{*}{ Moyenne } \\
\cline { 2 - 4 } & 14 jours & 21 jours & 28 jours & \\
\hline D0 & $51,563 a$ & $71,875 a$ & $75,000 a$ & $66,146 a$ \\
D1 & $59,75 a$ & $79,688 a$ & $81,250 a$ & $73,438 a$ \\
D2 & $56,250 a$ & $82,813 a$ & $84,375 a$ & $74,479 a$ \\
D3 & $54,688 \mathrm{a}$ & $82,813 a$ & $87,500 a$ & $75,000 \mathrm{a}$ \\
CV (\%) & $\mathbf{1 9 , 1 0 5 1 5}$ & $\mathbf{9 , 4 4 9 9 1 4}$ & $\mathbf{1 0 , 5 4 8 0 9}$ & $\mathbf{2 0 , 7 8 3 0 8}$ \\
\hline Pr> F & $\mathbf{0 , 7 7 1 4}$ & $\mathbf{0 , 1 8 3 7}$ & $\mathbf{0 , 2 5 9 3}$ & $\mathbf{0 , 4 4 4 9}$ \\
\hline
\end{tabular}

Les valeurs ayant la même lettre ne sont pas significativement différents au seuil de $5 \%$

Indice de vigueur: Le tableau 4 présente les valeurs moyennes des indices de vigueur de plants de manioc pour les différents traitements. Les parcelles fertilisées avec les traitements ont un taux d'accroissement variant de 12,65 à $14,59 \%$ de la première à la troisième semaine d'observation.

Tableau 4 : Moyennes des indices de vigueur des plants de manioc

\begin{tabular}{l|c|c|c|c}
\hline \multirow{2}{*}{ Traitements } & \multicolumn{3}{|c|}{ Indice de vigueur des plants } & \multirow{2}{*}{ Moyenne } \\
\cline { 2 - 4 } & 64 jours & 71 jours & 78 jours & \\
\hline T0 & $1,81 \mathrm{~b}$ & $1,99 \mathrm{c}$ & $2,127 \mathrm{~b}$ & $1,97 \mathrm{c}$ \\
T1 & $1,99 \mathrm{a}$ & $2,09 \mathrm{bc}$ & $2,33 \mathrm{a}$ & $2,14 \mathrm{~b}$ \\
T2 & $2,05 \mathrm{a}$ & $2,25 \mathrm{ab}$ & $2,37 \mathrm{a}$ & $2,23 \mathrm{ab}$ \\
T3 & $2,14 \mathrm{a}$ & $2,38 \mathrm{a}$ & $2,45 \mathrm{a}$ & $2,32 \mathrm{a}$ \\
\hline CV (\%) & $\mathbf{1 8 , 1 8 7 9}$ & $\mathbf{1 7 , 1 4 2 6}$ & $\mathbf{1 6 , 2 2 1 7}$ & $\mathbf{1 8 , 0 8 5 7}$ \\
\hline Pr> F & $\mathbf{0 , 0 0 0 7}$ & $<0,0001$ & $\mathbf{0 , 0 0 1 7}$ & $<0,0001$ \\
\hline
\end{tabular}

Les valeurs ayant la même lettre ne sont pas significativement différents au seuil de $5 \%$

Hauteurs de plants : Le tableau 5 présente les valeurs moyennes de hauteurs de plants de manioc de 64 à 78 jours après plantation des boutures pour les différents traitements. Les résultats entre les hauteurs sont très significatifs pour toutes les dates de mesure $(<0.0001)$.
Les plus petites tailles ont été observées sur les parcelles témoin (T0). Par contre, les plus grandes tailles des plants de manioc ont été sur les parcelles fertilisées avec le traitement T3 (Tableau 5).

Tableau 5 : Moyennes de hauteurs des plants de manioc

\begin{tabular}{c|c|c|c|c}
\hline \multirow{2}{*}{ Traitements } & \multicolumn{3}{|c|}{ Hauteur des plants } & \multirow{2}{*}{ Moyenne } \\
\cline { 2 - 4 } & 64 jours & 71 jours & 78 jours & $27,618 \mathrm{c}$ \\
T0 & $23,165 \mathrm{~b}$ & $27,271 \mathrm{~b}$ & $32,551 \mathrm{~b}$ & $33,138 \mathrm{~b}$ \\
T1 & $28,813 \mathrm{~b}$ & $31,628 \mathrm{~b}$ & $39,053 \mathrm{~b}$ & $42,453 \mathrm{a}$ \\
T2 & $37,488 \mathrm{a}$ & $43,203 \mathrm{a}$ & $46,668 \mathrm{a}$ & $45,075 \mathrm{a}$ \\
T3 & $39,313 \mathrm{a}$ & $47,598 \mathrm{a}$ & $48,397 \mathrm{a}$ & 39,70694 \\
CV (\%) & 40,51811 & 36,37741 & 38,52297 & $<0,0001$ \\
\hline Pr> F & $<0,0001$ & $<0,0001$ & $<0,0001$ &
\end{tabular}

Les valeurs ayant la même lettre ne sont pas significativement différents au seuil de $5 \%$ 


\section{DISCUSSION}

Avant l'épandage de fertilisants organique, les résultats chimiques du sol ont relevé un sol très pauvre en matière organique $(\mathrm{C}=7,3 \mathrm{~g} / \mathrm{kg}$ et $\mathrm{N}=0,3 \mathrm{~g} / \mathrm{kg})$, et mal décomposé $(\mathrm{C} / \mathrm{N}=24,3)$. Ces propriétés témoignent d'un très faible potentiel de fixation et d'échange d'éléments selon la somme des bases échangeables $(\mathrm{S}=0,23 \mathrm{Cmol} / \mathrm{kg}$ ) et du taux de saturation ( $\mathrm{V}=9,91 \%$ ). C'est un sol très acide ( $\mathrm{pH}$ eau $=4,2)$ et appartenant à la sous classe des Ferralsols (Akanza et Yao-Kouamé, 2011). Les résultats mettent en évidence une nette amélioration de la fertilité chimique du sol par les téguments. L'évolution $\mathrm{du} \mathrm{pH}$, du rapport $\mathrm{C} / \mathrm{N}$, de la CEC et de la somme des cations en témoigne. L'accroissement de chacune des caractéristiques est d'autant plus marqué que la dose de téguments est forte. Les téguments de fèves de cacao sont riches en carbone total, en azote total, en calcium échangeable, en magnésium et en potassium échangeables. Le fumier de téguments apporté a produit des effets significatifs sur les paramètres chimiques des sols dégradés de la zone d'Ahoué par l'amélioration des CEC, de la matière organique, de la somme des bases échangeables, du taux de saturation en bases et du pH eau des sols fertilisés et ce, d'autant plus avec le traitement T3. En effet, avec leur décomposition dans le sol, ces téguments assurent une grande disponibilité en éléments nutritifs pour les plants de manioc. Ces résultats sont en accord avec ceux de Useni et al. (2013), Djeke et al. (2011) et MoyinJesu (2007) qui ont montré, respectivement, que la décomposition des résidus végétaux a relevé les niveaux $\mathrm{du} \mathrm{pH}$ et des nutriments du sol. L'accroissement de $\mathrm{pH}$ du sol observé dans les parcelles traitées traduit une baisse de l'acidité du sol. Avec le traitement T3, l'acidité du sol passant de 4,2 à 5,8, serait liée aux ions $\mathrm{Ca}^{2+}$ et $\mathrm{Mg}^{2+}$, qui, en dépit, de leur prélèvement par les plants, sont en quantités suffisantes pour neutraliser les ions responsables de l'acidité du sol. L'augmentation de la CEC par l'apport des téguments de fèves de cacao pourrait s'expliquer par le fait que ces amendements ont affecté les phénomènes de fixation des cations échangeables sur le complexe absorbant des sols (Abobi et al., 2014). Selon les travaux de Djeké et al. (2011), étant donné que le pH permet de définir l'état du complexe adsorbant, notamment, le taux de saturation en bases (Duchaufour, 1997), son amélioration par l'apport de téguments des fèves traduit aussi celle du taux de saturation en bases. Les résultats, ainsi obtenus, sont parfaitement concordants pour authentifier l'hypothèse selon laquelle la matière organique, sous forme de téguments de fèves de cacao, est l'un des véritables moteurs de l'amélioration de la fertilité chimique du sol. Elle s'emploie à forte dose (Adden, 2004). L'analyse des données établit en tête de classement que le traitement $\mathrm{T} 3$ semble réaliser le meilleur résultat. Cette observation certifie que l'apport d'engrais organiques à fortes doses est indispensable pour maintenir ou accroître conjointement les stocks de carbone total et d'éléments nutritifs des sols (Thuriès et al., 2000). Les résultats de l'étude montrent que le taux de levée de la variété de manioc (Improved African Cassava) est plus important lorsqu'on s'éloigne de la période de semailles. L'analyse statistique ne révèle aucune différence significative entre les moyennes du taux de levée, pour les différents traitements $(P>0.05)$. Les résultats non significatifs voudraient dire que les premiers germes proviendraient des réserves nutritives des boutures mises en sol. Ceci est en accord avec les travaux de Egle (1992), qui indiquent que pendant la période de dormance, la plante utilise sa propre réserve nutritionnelle. Les moyennes des taux de levée variant de l'ordre de 60 à $75 \%$ pour les 3 dates de mesures, mettent en cause la qualité des boutures de manioc utilisées. Les résultats obtenus lors de cette étude corroborent avec ceux de Egle (1992), qui indiquent que le taux de reprise est régulièrement conditionné par la bonne qualité de bouture, et est l'une des conditions essentielles de départ pour assurer un peuplement homogène d'une parcelle de manioc. Les résultats obtenus de la vigueur des plantes de manioc avec les effets de doses croissantes de téguments de fèves de cacao sont très statistiquement différents $(P<0,0001)$ par rapport au niveau de contrôle TO et contrairement au taux de levée. Selon les travaux de Useni et al. (2013) et Kouadio (2007), l'efficience des résidus de sous-produits de cacao, peut être probablement attribuée à leur décomposition pour la mise à la disposition des éléments nutritifs. Ceux-ci favorisent la rigidité des tissus végétaux et, par conséquent, la vigueur des plantes. Les valeurs moyennes de l'indice de vigueur et de la hauteur des plants de manioc ont été plus grandes avec la dose T3 (20t/ha). Les résultats très significatifs avec le traitement $T 3$, voudraient dire qu'un apport important de fertilisants organiques accélère la croissance des plants de manioc. Cette croissance rapide s'expliquerait par la forte activité biologique suite à l'apport important de téguments de fèves de cacao, ce qui favoriserait la décomposition rapide de ces produits pour la mise à disposition des 
éléments nutritifs dans le sol. En accord avec les travaux de Useni et al. (2013), tout apport organique provoque la stimulation de la biomasse microbienne, compartiment vivant de la matière organique du sol (Girard et al. 2005). L'action des matières organiques sur les propriétés biologiques consiste essentiellement à l'hébergement d'organismes vivants du sol et constitue une source nutritive de la microfaune du sol (Calvet, 2003). Les micro-organismes influent sur les propriétés chimiques du sol, dans un sol bien

\section{CONCLUSION ET PERSPECTIVES}

Cette étude, menée sur les Ferralsols à Ahoué, au SudEst de la Côte d'Ivoire, a montré que l'utilisation de téguments de fèves de cacao comme engrais organique, est une des solutions pour la restauration de la fertilité des sols dégradés. L'utilisation des téguments a eu un effet bénéfique sur la fertilité chimique du sol et les paramètres de croissance mesurés du manioc. L'apport de 20 tha $^{-1}$ de téguments, réalise le meilleur compromis aussi bien entre les paramètres chimiques du sol et les paramètres de croissance du manioc. L'apport ou la restitution de matière organique au sol constitue à la fois un amendement et un apport alimentaire pour la plante. La matière organique, une fois incorporée au sol, subit une série de

\section{REFERENCE BIBLIOGRAPHIQUE}

Abobi A. H. D., Angui T. K. P., et Kouadio Y.J., 2014. Influence de la fertilisation à base des coques de cacao sur les paramètres chimiques d'un ferralsol et sur la croissance du maïs à Oumé Côte d'Ivoire. Appl. Biosci. Journal of Applied Biosciences 82: pp.7359 - 7371.

Adden A. K., 2004. Évaluation quantitative des composts de biomasses diverses et leur phytotoxicité. Mémoire d'Ingénieur Agronome. Université de Lomé, $44 \mathrm{p}$.

Akanza P.K. et Yao-Kouamé A., 2011. Fertilisation organo-minérale du manioc (Manihot esculenta Crantz) et diagnostic des carences du sol. Journal of Applied Biosciences, 46 : pp.3163-3172.

Angui P. K. T., Tié B. T., Tamia A. J., et Yoro G., 2005. Typologie des sols de Côte d'Ivoire et leur utilisation. Bioterre et Rev. Inter. Sci. de la vie et de la terre. Vol $5 \mathrm{~N}^{\circ} 1$. pp. 1-16.

Ayanlaja S.A. et Sanwo J.O., 1991. Management of soil organic matter in farming systems of the lowland humid trop of West Africa. Soil Technol., 4 : pp. 265-279. équilibré, la biomasse microbienne se comporte comme un réservoir d'éléments minéraux : elle les maintient (bio immobilisation temporaire) dans les horizons supérieurs du sol, les protège du lessivage, et les restitue progressivement aux plantes (Ros et al., 2006). Les téguments de fèves de cacao, apportés aux sols pauvres et acides ont fourni des éléments nutritifs nécessaires pour l'alimentation et la croissance des plants de manioc.

transformations qui, sous l'action des micro-organismes et sous l'influence du milieu, la décomposent en humus et la minéralisent. Ces transformations étant très bénéfiques pour le sol, les apports de matières organiques devraient se réaliser, en principe, tous les ans, pour des agriculteurs soucieux de la préservation de leur capital sol. La valorisation agronomique des téguments de fèves de cacao mérite d'être approfondie car leur utilisation pourrait être une solution dans la gestion de la fertilité du sol pour une agriculture durable. Dans le futur, il serait intéressant de préciser la dose optimale des téguments de fèves de cacao et de déterminer leurs effets sur les sols en combinaison avec les engrais minéraux.

Bakayoko S., Soro D., N'Dri Be., Kouadio K.K.H., Tschannen A., Nindjin C., Dao D., et Girardin O., 2013. Étude de l'architecture végétale de 14 variétés améliorées de manioc. Journal of Applied Biosciences, 61: pp. 4471- 4477.

Boli Z. et Roose E., 2000. Rôle de la jachère de courte durée dans la restauration de la productivité des sols dégradés par la culture continue en savane soudanienne humide Du NordCameroun.In : Floret Ch. et Pontanier R., eds. La jachère en Afrique tropicale. Paris: John Libbey Eurotext, pp. 149 - 154.

Bremner J. M. and Mulvaney C., 1982. Total nitrogen and total P. In: A. L. Page, R. H. Miller, D. R. Keeney (eds). Methods of Soil Analysis. Part 2: Chemical and Microbiological Properties. Agron. Series 9. American Society of Agronomy. Madison, Wisconsin: pp $403-430$.

Calvet R., 2003. Le sol, propriétés et fonctions. Tome 2. Phénomènes physiques et chimiques, applications agronomiques et environnementales. Edts France Agricole, Dunod, $471 \mathrm{p}$. 
Cattan P., Letourmy P., Zagre B., Minougou A. et Compaoré E., (2001). Rendement de l'arachide et du sorgho en rotation sous différents itinéraires techniques au Burkina Faso. Cahiers Agricultures, $10: 159-172$.

Dabin B., 1967. Méthode Olsen modifiée. Cahier ORSTOM. Pédologie, pp. 3 - 5.

Djeké M.D., Kouassi P., Tehua A., et Kouadio J.Y., 2011. Décomposition des broyats de coques de cacao dans les sols ferralitiques de la zone d'Oumé, Centre-Ouest de la Côte d'Ivoire : effets sur les caractéristiques chimiques des sols. Faculté des Sciences de l'Agriculture et de l'Alimentation, Québec (Canada) , $10 \mathrm{p}$.

Duchaufour P., 1997. Abrégé de pédologie. Sol, végétation, environnement. 5eéd. Paris: Masson. $12 \mathrm{p}$.

Egle K., 1992. Étude de la variabilité des composantes du rendement du manioc (Manihot Esculenta Crantz var. 312-524) en fonction de la fertilité du sol. Mémoire d'ingénieure agronome. École Supérieure d'Agronomie de Lomé, $122 \mathrm{p}$.

FAO, 2003. Gestion de la fertilité des sols pour la sécurité alimentaire en Afriquesubsaharienne, Rome, ISBN 92-5-204563-5.

Girard, M.C., Walter C., Remy J.C., Berthelin J., et Morel J.L., 2005. Sols et environnement. Édition Dunod, Paris, $800 \mathrm{p}$.

Hauser S., 2013. Natural Resource management in cassava and Yam production systems. R4D Review - Best practice, Issue 9. Ibadan, Nigéria, IITA : pp. 109-127.

Kouadio K. P., 2007. Effet du broyat de coquillages d'huître et d'escargot sur la croissance et la production du soja. Mémoire de DEA. Université Nangui Abrogoua, $33 p$.

Kouakou J. K., Yapi A., Alui A.K., Akotto F.O., et Yaokouame A., 2016. Pédopaysage et distribution de Imperata cylindrica (L.) P. Beauv. (Poaceae) dans deux Agro-écosystèmes de la Côte d'Ivoire : Abidjan et Bouaké. International Journal of Innovation and Scientific Research ISSN 2351-8014 Vol. 22 No. pp. 238-249.

Moyin-Jesu E. I., 2007. Use of plant residue for improving Soil fertility, pod nutrients, root growth and pod weigth of okra (Abelmoschus esculentrum L). Bioresour. Technol.98, pp.2057 - 2064.

N'Goran A., Gnahoua G. M., Oualou K., et Pity B., 1997. Évolution de la fertilité d'un sol au cours de quatre ans de culture suite à une jachère arborée de six ans. Cas d'une zone de forêt humide en Côte d'Ivoire. In: Amélioration et gestion de la jachère en Afrique de l'Ouest. ORSTOM, Paris, France, pp. 101-106.

N'zué B., Zohouri G. P., Yapi-Gnahoré V., et Djédji C., 2005. Bien cultivar le manioc en Côte d'Ivoire. Fiche technique. CNRA-CTA, Abidjan, $4 \mathrm{p}$.

Olsen S. R. and Sommers L. E., 1982. Phosphorus. In: A. L. Page, R. H. Miller and D. R. Keeney (eds). Methods of Soil Analysis. Part 2: Chemical and Microbiological Properties. Second Edition. Agronomy 9. American Society of Agronomy. Madison, Wisconsin : pp. 403 - 427.

Ros M., Klammer S., Knapp B., Alchberger K.,et Insam H., 2006. Long-term effect of compost amendent of soil on functional and structural diversity and microbial activity. Soil Use and Management, 22 : pp. 209-218.

Sangare O., Bastos A.D.S., Marquardt O., Venter E.H., Vosloo W., et Thomson G.R., 2001. Molecular epidemiology of serotype $O$ foot and mouth disease virus with emphasis on West and South Africa. Virus Genes 22: pp. 345 351.

Thuriès L., Arrufat A., Dubois M., Feller C., Hermann P., Larré-Larrouy M. C., Martin C., Pansu M., Rémy J. C. et Viel M., 2000. Influence d'une fertilisation organique et de la solarisation sur la productivité ma

Useni S.Y., Chukiyabo K.M., Tshomba K.J., Muyambo M.E., Kapalanga K.P., Ntumba N.F., Kasangij K.P., Kyungu K.A., Baboy L.L., Nyembo K.L., et Mpundu M.M., 2013. Utilisation des déchets humains recyclés pour l'augmentation de la production du maïs (Zea mays L.) sur un ferralsol du Sud-Est de la RD Congo. Journal of Applied Biosciences 66: pp. $5070-50811$.

Walkley A. et Black I.A., 1934. An examination of the Degtjareff method for determining soil organic matter and a proposed modification of the chromic acid titration method. Soil Sciences. 37 : pp. 29 - 38.

Yemefack M. et Nounamo L., 2000. Dynamique des sols et durée optimale des jachères agricoles au Sud-Cameroun. In: Floret C. Pontanier R., eds. La jachère en Afrique tropicale. Paris, pp. 135-141. 